\title{
Robotized System for Bone Drilling and Cutting in Orthopedic Surgery
}

\author{
George Boiadjiev $^{1, \text { a }}$, Vladimir Kotev ${ }^{1, \mathrm{~b}}$, Kazimir Zagurski ${ }^{2, \mathrm{c}}$, Kamen Delchev ${ }^{1, \mathrm{~d}}$, \\ Tony Boiadjiev ${ }^{3, e}$ and Rumen Kastelov ${ }^{4, f}$ \\ ${ }^{1}$ Institute of Mechanics - BAS, Sofia, Bulgaria \\ ${ }^{2}$ Institute of Rbobtics and System Engineering - BAS, Sofia, Bulgaria \\ ${ }^{3}$ Institute of Information and Communication Technologies - BAS, Sofia, Bulgaria \\ “University Hospital for Emergency Medicine "Pirogov" - Sofia, Bulgaria

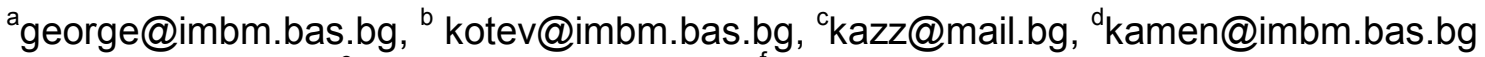 \\ etonyboiadjiev@mail.bg, ${ }^{\mathrm{f}}$ rkastelov@yahoo.com
}

Keywords: Control; Robotics; Orthopedic surgery.

\begin{abstract}
Bone drilling and cutting manipulations are widespread in the orthopedic surgery. In free hand performance of them some errors such as an inaccurate penetration and dilate of bone hole, overheating, harm soft tissues could be occurring. The goal of this study is to design and develop control concept for a bone drilling and cutting robotized system. The proposed orthopedic system consists of two executive modules for drilling and cutting and common control panel. The control algorithms and programs for drilling have done and given in the paper. We are developing the control algorithms for cutting manipulation.
\end{abstract}

\section{Introduction}

Bone drilling and cutting manipulations are often performed in the orthopedic surgery. Total knee arthroplasty (TKA) and Total hip arthroplasty (THA) as well as bone fractures require cutting and drilling manipulations [1]-[6]. The successful execution of bone drilling and cutting requires a high level of precision, dexterity and experience. In free hand drilling and cutting some problems may occur related to the performance of operations. Examples include the possibility of breaking the blood vessels if the drill bit goes through the bone, or widening of hole diameter by the oscillations, or rough surface of bone in cutting operations. Also, bone overheating is an important problem [6]-[13]. Robotics can enhance surgery through improved precision, stability, quality of performed manipulations and dexterity [1]-[5], [7]-[10]. With incorporation of computed tomography, magnetic resonance images and new algorithms operative plans and the execution of these plans are becoming more precise and accurate. The ultimate aim of this technology is to reduce patients' pain and improve functional outcomes [1]-[3], [7], [8], [14]. Some of the first developed orthopedic surgery robots were Robodoc, Caspar [1]-[3], [14], Acrobot [1], [2], [14] . Many surgical operations have been performed all over the word by MAKO, and Da Vinci robots [1], [2], [14]. However, these computer assistive orthopedic surgery robotized systems are expensive and require special maintenance [1], [2], [14]. They are not affordable for most hospitals. During the past decade small size, special and low cost robot has been developing [2], [4], [5], [11]-[16]. Several studies refer to the development of bone-mounted robots such as Praxiteles [5], MARS [16], or hand-held robots [2], [11]-[13].

The aim of the present paper is to develop a hand-held bone drilling and cutting robotized orthopedic system. It is intended to be applicable for TKA and THA operations as well as fracture treatment. The designed orthopedic system consists of two executive modules for automatic bone drilling and cutting respectively and a control module. In the previous authors' research [11]-[13], [16] an orthopedic drilling robot (ODRO) was developed. We are going to improve the mechanical module of ODRO and to develop a bone sawing module. A common control panel will be designed. 


\section{Mechanical Design of the System}

Improvement of ODRO. The ODRO robot is developed to improve patient's safety by removing the subjective factor and avoid the problems caused by hand bone drilling and increase manipulation accuracy. Also, it is intended to identify some parameters of bone drilling process, such as resistant force due to variable bone density, appropriate mechanical torque during the manipulation, and linear speed. It has one translation and one revolute joint with co-linear axes. The first motor of ODRO is brushless DC Maxon [17] for drilling motion. The second motor is a linear actuator Haydon Switch \& Instrument 43000-17 [18]. ODRO has got the following functional parameters: working zone: from 0 to $105 \mathrm{~mm}$, precision $0.5 \mathrm{~mm}$, rotation speed from 0 to $900 \mathrm{rpm}$ and translation speed from 0 to 9 $\mathrm{mm} / \mathrm{s}$. Geometrical characteristics of ODRO are: max diameter $64 \mathrm{~mm}$ and length $365 \mathrm{~mm}$. In order to decrease length and increase of working zone of the modified robot we suggest the axis of motors to be parallel, unlike of these up to now. On Fig.1 it is shown a 3D model of the improved robot ODRO-M. Regarding to the parallel structure the working zone becomes $121 \mathrm{~mm}$, the length becomes $220 \mathrm{~mm}$ and height becomes $110 \mathrm{~mm}$. Fig.1.b. shows 3D model of the whole assembly of the bone drilling robot ODRO-M.

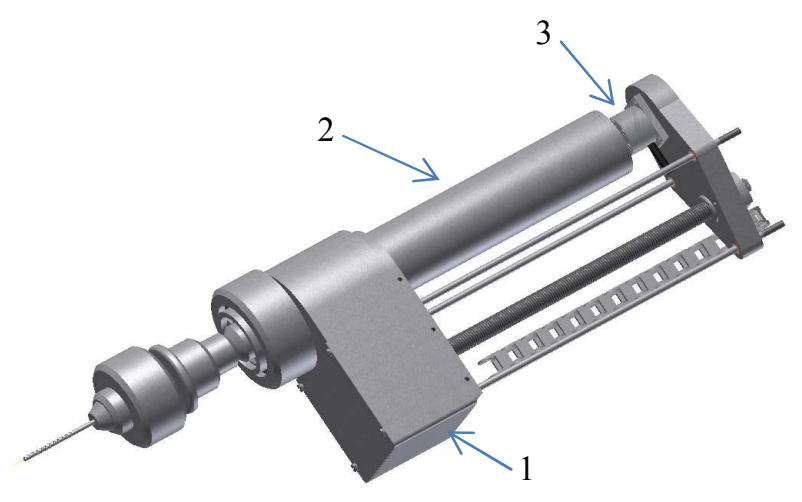

a

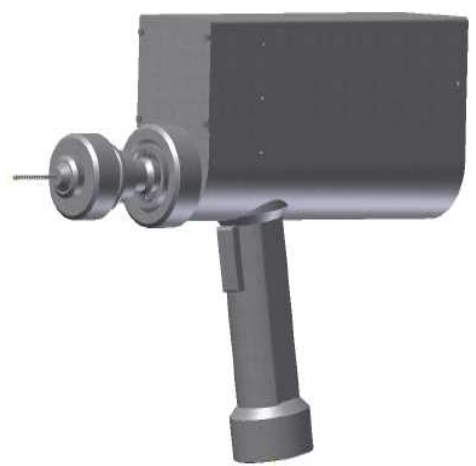

$\mathrm{b}$

Fig.1. (a) Assembly of the linear motor-1, executive module -2 and force sensor -3 , (b) ODRO - M.

Design of an orthopedic cutting robot OCRO. A design concept for the development of a bone cutting robot OCRO is worked out. The module is intended to perform the cutting process with appropriate thrust force and feed rate due to variable bone density. It can automatically set the cutting conditions in order to maintain low temperature during the cutting process execution. The robot has to be sterile, reliable, with simple construction, small size and weight. We are using the same scheme like ODRO-M which is shown on Fig.1.a.

Based on the date of literature, companies' catalogues and simulation we have selected the main components of the hand-held bone cutting robotized prototype OCRO. These are:

- Orthopedic oscillating saw attachment - RJ1091 [19],

- Brushless DC motor MAXON [20]. The motor is equipped by servo controller/driver 1-Q-CE and amplifier DEC 50-5 [21] Fig.2 (a).

- Linear motor MB0801 [22]. Five phase stepper motor with embedded screw for linear motion.

- Small and lightweight LMB-A force sensor for measurement the thrust force [22]. 


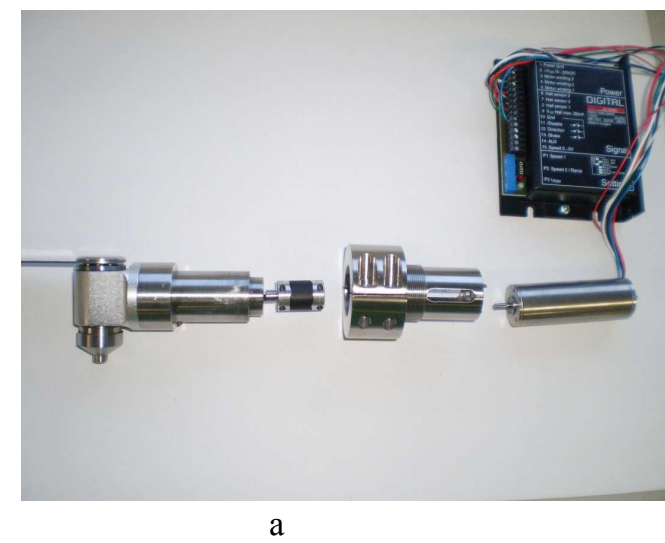

Fig. 2. (a) cutting assembly, (b) bone cutting prototype OCRO.

\section{Control Concept}

ODRO's control algorithms will be implemented in the improved bone drilling module ODRO-M. In order to test ODRO's performance and control algorithms many experiments were done. In [11]-[13] some experiential results are published. We are developing control algorithms and programs for a bone cutting module OCRO. A common control panel for the both executive modules will be design.

ODRO's Control Algorithms. Control system of ODRO gives information about the drilling process execution in real time, for successful end of the task as well as for emergency situation. The control block has terminals for connection with PC. They give a possibility to re-program the software, which is recorded in the controllers. Controllers can change and update the programs and to transfer the information between the sensors and PC while the drilling is executed in real time.

The control algorithms are executed in the specific for the controllers specialized program language TMCL-IDE. The commands can be executed immediately after their input (direct regime) or the program can be downloaded in the controller for autonomous execution (stand-alone regime). For every cycle the program recognize the current state, executes the corresponding algorithm and takes a decision depending on preliminary determined criteria for going to the next state.

An algorithm for current calculation of the "next target position" for the stepper motor is realized depending on the force sensor data. The actual force sensor data are considered in comparison with the previous given values. The last reflects to the safety requirements that the exceeding of a given limit value is not allowed.

An algorithm for identification of the resistant force value during the drilling process is realized. This value is in relation with the objects manipulation characteristics and used drill bit. The obtained value is used for taking the decision for completion of the bone cortex drilling.

The control algorithms work in two regimes: drilling to reach a preliminary desired depth and drilling through the whole bone. In the last case the control algorithm allows breaking the drilling process automatically when the cutting surface of the drill bit reaches the bone marrow. Then the surgeon has to make a decision to go on with the drilling of the second cortex or to terminate the task. The task is completed when the cutting surface reaches the soft tissue behind the bone and the drilling stops automatically.

After the settings of the working task have been given, the following sequence of acts occurs: going through reference position, positioning at start position, stopping and waiting for control signal by the start-button for beginning the task execution, searching for a contact and transferring the control signal for starting the rotation. In the "preliminary given length of depth drilling" mode when the given depth is achieved, the rotation stops and the executive module (translation) returns back to "Reference Position". Then a check for missed steps of the motor takes place and as a result a decision is made to convert the state into "Drill Ready" or "Drill Again". 
In "bone drilling" mode the force identification is made. The obtained value is used for taking the decision for completion of the bone cortex drilling. After drilling out the first bone cortex the translation is stopped automatically. For going on the program the controller is waiting to receive the

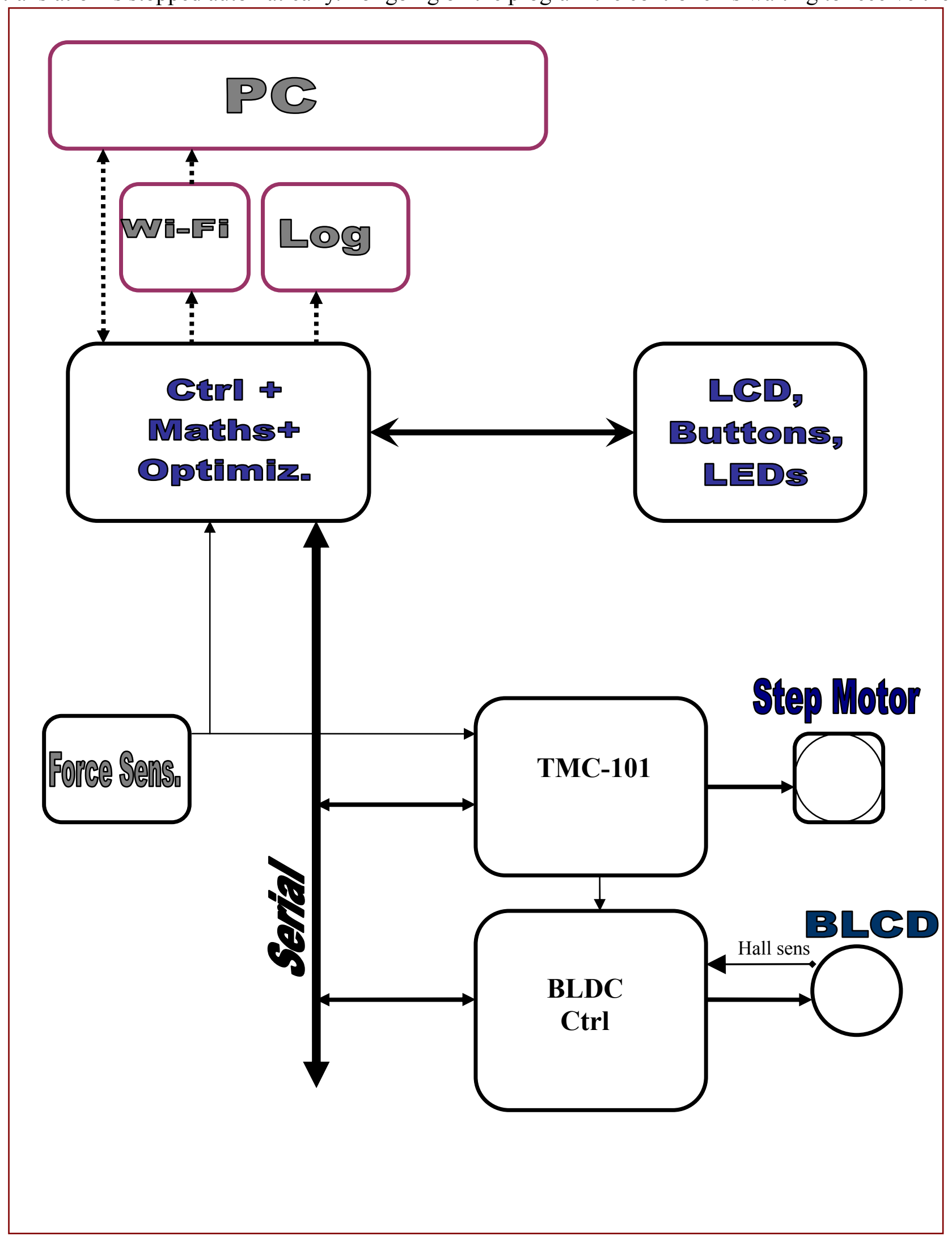

Fig.3. The block diagram of multi-controller system. 
corresponding signal by the surgeon who presses the start button again. After drilling out of the second cortex, the rotation is stopped automatically and the machine converts to the state "Drill Ready", reaching the "Start position".

Before drilling start the robot is doing "self-test" to specify its state and confirm a readiness for work. The "self-test" includes checking of the working zone, force sensor, start button and reference position. Such a "self-test" is important for safe execution of drilling.

OCRO's Strategies for Control. Determination of the following cutting conditions, such as thrust force, torque, speed, feed rate, depth of cut are essential for the quality performance of the bone cutting process. In order to maintain lower temperature and smoothness of bone surface the cutting conditions should automatically change. It is known that feed rate and depth of cut significantly change the cutting force [5]-[13].Thus, an appropriate control of the thrust force will allow avoidance of a sudden stop of the cutting process caused by friction arising for the sake of uncontrollable thrust force.

In order to ensure a high-level control of both drilling and cutting modules ODRO-M (see Fig.1.b) and OCRO (see Fig.2.b) a multi-controller system is proposed. The block diagram of the proposed multi-controller system is shown in Fig. 3. The "master controller" provides functions for control of the system, mathematical calculations, force sensor data acquisition and optimization of the drilling, and cutting processes as well as functions for interface control. The "master controller" also provides "Serial" and "Wi-Fi" connections to a Personal Computer for its reprogramming and data processing. It has to be mentioned that additional Step Motor and BLDC (Brushless DC Motor) controllers could be connected to the Serial Bus of the system.

\section{Summary}

We have been working on development of both bone cutting and drilling module with parallel structure of motor axis and with a common control unit. The usage of such a robotized drilling/cutting system can shorten the duration of the orthopedic surgery operations avoiding the necessity of replacement of the drill and saw attachments of the standard medical drill machines during the operation. We are developing control algorithms and a common control panel for these two executive modules. The mechanical structure of ODRO is improved in the sense of decreasing length and increasing its working zone. In order to make it lighter we are going to use titanium alloy instead of stainless steel. This way the weight will be decreased about 40 percent. It will be much convenient for surgeon's practical work. The design concept of a bone cutting saw OCRO has done. The saw is intended to perform cutting operations with preliminary setting of depth and stop automatically after the cutting process is completed. Cutting conditions will automatically change in accordance with bone density. We are going to develop multiprocessing control system algorithms and programs.

\section{Acknowledgment}

The financial support of Bulgarian Science Fund, Bulgarian Ministry of Education, Youth and Science, Grant FFNNIPO_12_01547 is acknowledged.

\section{References}

[1] J. Rosen, B. Hannaford, R. Satava, Surgical Robotics. Springer Science+Business Media, LLC 2011.

[2] P. Gomes, "Surgical robotics: Reviewing the past, analyzing the present, imagining the future". Robotics and Computer-Integrated Manufacturing, vol. 27, 2011, pp. 261-266.

[3] N. Sugano,"Computer-assisted orthopedic surgery", Journal of Orthopedic Science, vol.8, 2003, pp.442-448. 
[4] W. Saensupho and J. Suthakorn, "A Novel Conceptual Design on Robotic Bone-Cutting Device Based on Kinematic Analysis: Toward Development of A Novel Robot-Assisted Total Knee Replacement Surgical System", 2011, International Conference on Computer Control and Automation (ICCCA 2011), pp. 139-142.

[5] C. Plaskos, P. Cinquin, S. Lavalle' e and A. Hodgson, "Praxiteles: a miniature bone-mounted robot for minimal access total knee arthroplasty", Int. J. Medical Robotics and Computer Assisted Surgery, vol.1 (4), 2005, pp. 67-79.

[6] N. Sugita, T. Osa and M. Mitsuishi,"Analysis and estimation of cutting-temperature distribution during end milling in relation to orthopedic surgery", Medical Engineering \& Physics, vol.31, 2009, pp.101-107.

[7] W. Lee and Ching-Long Shih, "Control and breakthrough detection of a three-axis robotic bone drilling system," Mechatronics, vol. 16, pp. 73-84, 2005.

[8] Z. Taha, A. Salah, and J. Lee, "Bone Breakthrough Detection for Orthopedic Robot-Assisted Surgery", APIEMS 2008 Proc. of the 9th Asia Pasific Industrial Engineering \& Management Systems Conf., 2742-2746, 2008.

[9] M. Tsai, M. Hsieh and C. Tsai,'Bone drilling haptic interaction for orthopedic surgical simulator", Computers in Biology and Medicine, 37, pp. 1709-1718, 2007.

[10] M. Hsieh, M. Tsai, and Y. Yeh, An amputation simulator with bone sawing haptic interaction, Biomed. Eng. Appl. Basis. Comm., 2006, (October), vol. 18, pp. 229-236.

[11] G.Boiadjiev, T.Boiadjiev, Vl. Vitkov, K.Delchev, R. Kastelov, K.Zagurski, ’Robotized System for Automation of the Drilling in the Orthopedic Surgery. Control Algorithms and Experimental Results “. In Proceedings of the 9th IFAC Symp. on Robot Control SYROCO'09, Gifu, Japan, 2009, pp.633-638.

[12]T. Boiadjiev, K. Zagurski, G. Boiadjiev,K. Delchev,Vl. Vitkov, I.Veneva, R. Kastelov, "Identification of the Bone Structure during the Automatic Drilling in the Orthopedic surgery". $J$. of Mechanics Based Design of Structures and Machines, vol. 39, pp. 285-302, 2011.

[13] G.Boiadjiev, K.Zagurski, T.Boiadjiev, K.Delchev, R. Kastelov and Vl.Kotev, Robot application in orthopedic surgery: drilling control, GSTF Jour. Eng. Technology, vol.1 (1), June 2012, pp.125-130.

[14] M. Toole, K. Marouf, D. Kerr, M. Gooroochurn and M. Vloeberghs, "A methodology for design and appraisal of surgical robotic systems", Robotica (2010), 8, pp. 297-310. Cambridge University Press 2009

[15] Shoham M., Burman M., Zehavi E., Joskowicz L., Batkilin E. and Y. Kunicher. Bone-Mounted Miniature Robot for Surgical Procedures: Concept and Clinical Applications, IEEE Transactions on Robotics and Automation, 19,(5), pp: 893-901, 2003.

[16] Patent No 1053 / 24.07.2008, BG.

[17] Maxon, motor catalogue 2012/13.

[18] http://www.haydonkerk.com/LinearMotionProducts/StepperMotorLinearActuators/tabid/66/De fault.aspx

[19] http://whruijin.en.made-in-china.com/product/feDmQMwGZNWd/China-Orthopedic-Oscillatin g-Saw-Attachment-RJ1091-.html

[20] Maxon, motor catalogue 2012/13, pp. 177. 
[21] Maxon motor catalogue 2012/13, pp. 239.

[22] www.kssballscrew.com/us/pdf/catalog/vol.25.0_MoBo_MB_A3.pdf

[23] http://www.kyowa-ei.co.jp/eng/product/sensors/loadcell/lmb_a.html 